\title{
Asymptotic Expansion Method with Respect to Small Parameter for Ternary Diffusion Models
}

\author{
Marek Danielewski ${ }^{1} \cdot$ Henryk Leszczyński ${ }^{2} \cdot$ Anna Szafrańska $^{3}$
}

Received: 13 July 2016 / Revised: 10 March 2017 / Accepted: 17 March 2017 / Published online: 12 April 2017

(C) The Author(s) 2017. This article is an open access publication

\begin{abstract}
Ternary diffusion models lead to strongly coupled systems of PDEs. We choose the smallest diffusion coefficient as a small parameter in a power series expansion whose components fulfill relatively simple equations. Although this series is divergent, one can use its finite sums to derive feasible numerical approximations, e.g. finite difference methods (FDMs).
\end{abstract}

Keywords Diffusion · Mass conservation - Difference scheme $\cdot$ Asymptotic series expansion $\cdot$ Small parameter

\section{Mathematics Subject Classification $35 \mathrm{~K} 57 \cdot 35 \mathrm{C} 20$.} $65 \mathrm{M} 06$

\section{Introduction}

The subject of divergent series dates back to the distant past and is linked with the names of outstanding mathematicians such as Euler, Poincaré, Borel, Padé, and Birkhoff.

Anna Szafrańska

aszafranska@mif.pg.gda.pl

Marek Danielewski

daniel@agh.edu.pl

Henryk Leszczyński

hleszcz@mat.ug.edu.pl

1 Faculty of Materials Science and Ceramics, AGH

University of Science and Technology, 30 Mickiewicza Av., 30-059 Krakow, Poland

2 Institute of Mathematics, University of Gdańsk, Wit Stwosz Street 57, 80-952 Gdańsk, Poland

3 Department of Differential Equations and Mathematics Applications, Gdańsk University of Technology, G. Narutowicz Street 11/12, 80-233 Gdańsk, Poland
For more details and additional references we refer to articles by Tucciarone [1] and Ferraro [2]. The idea of the asymptotic series comes from Poincaré, who introduced the definition of an asymptotic expansion. Nowadays, the importance of methods which are based on asymptotic expansions of solutions in small or large parameters series has grown considerably in many branches of physical, chemical, biological and engineering sciences. We mention several examples among a wide range of applications, and the list is not exhaustive. The fundamental problem of reconstruction of physical observables, based on divergent power series expansions, has been considered in many areas of quantum physics. Dyson already showed in his pioneering work [3] that expansions in quantum electrodynamics diverge factorially. After that, it was found that divergent perturbation expansions of quantum physics are very common $[4,5]$. This observation led to a substantial amount of research works in various areas of quantum physics, for example in quantum field theory [6-8] or in quantum mechanics $[9,10]$. The asymptotic analysis of singularly perturbed problems is performed in the monograph [11], based on expansions of analytical solutions in the power series with respect to a small parameter. In the light of this approach there are discussed models of populations, epidemiological problems, classical models of fluid dynamics and many others. The paper [12] deals with the method of asymptotic expansions of solutions to a singularly perturbed system of integro-differential equations in epidemiology with two small parameters. Asymptotic solutions are constructed by the Tikhonov-Vasil'eva method of boundary functions.

This work concerns a system of diffusion equations. When the number of components exceeds two, the term 'interdiffusion' or 'cross-diffusion' is often used, see [13] in material sciences and [14] in life sciences. The drift in 
each of the PDEs depends on the gradient of all other variables, so these models exhibit the phenomenon of crossdiffusion. This makes the PDE system strongly coupled. In addition, the coefficients depend on the unknown functions and the parabolicity of this system is conditional, namely it can be proved only if solutions remain in an admissible set. The invariance of this set is connected to mass conservation laws. Any violation of this property leads to serious analytical and computational problems. We overcome these problems by constructing conservative numerical schemes, i.e. preserving a discrete version of mass. In spite of mathematical and numerical difficulties, three dimensional interdiffusion problems are still of interest. Interdiffusion becomes an important topic in electrochemistry and biochemistry, e.g. molecular channels (nano-channels) [15].

\subsection{Preliminaries}

Let $\Omega$ be a bounded domain in $\mathbb{R}^{n}$ with a smooth boundary $\partial \Omega$. The closure of $\Omega$ is denoted by $\bar{\Omega}$. In this manuscript, we investigate the system of diffusion equations

$\left\{\begin{array}{l}\frac{\partial u}{\partial t}=\nabla \cdot\left(D_{1} \nabla u-u v^{D}\right) \\ \frac{\partial v}{\partial t}=\nabla \cdot\left(D_{2} \nabla v-v v^{D}\right) \\ \frac{\partial w}{\partial t}=\nabla \cdot\left(D_{3} \nabla w-w v^{D}\right)\end{array}\right.$

on $[0, T] \times \Omega$, where $D_{1}, D_{2}, D_{3}>0$ and $v^{D}$ is the drift velocity given by

$v^{D}=\nabla\left(D_{1} u+D_{2} v+D_{3} w\right)$.

The system (1) is considered with the initial conditions

$u(0, x)=u_{0}(x), \quad v(0, x)=v_{0}(x), \quad w(0, x)=w_{0}(x)$ for $x \in \bar{\Omega}$,

and the Neumann boundary conditions

$\frac{\partial u}{\partial \mathbf{n}}=\frac{\partial v}{\partial \mathbf{n}}=\frac{\partial w}{\partial \mathbf{n}}=0$ on $\partial \Omega$.

where $\mathbf{n}$ denotes the outward normal vector to the boundary $\partial \Omega$.

Our analysis of the strongly coupled system (1) starts from the simplest case $w \equiv 0$. Consider two chemical substances and their normalized densities $u, v \geq 0, u+v \equiv 1$ described by the system of differential equations

$\left\{\begin{array}{l}\frac{\partial u}{\partial t}=\nabla \cdot\left(D_{1} \nabla u-u \nabla\left(D_{1} u+D_{2} v\right)\right) \\ \frac{\partial v}{\partial t}=\nabla \cdot\left(D_{2} \nabla v-v \nabla\left(D_{1} u+D_{2} v\right)\right)\end{array}\right.$

It is clear that the assumptions on initial functions $u_{0}, v_{0} \geq 0$ and $u_{0}+v_{0} \equiv 1$ imply $u, v \geq 0, u+v \equiv 1$. Indeed, consider new dependent variables $p, q$ defined as linear combinations of the functions $u, v$ :

$p=u+v, \quad q=D_{1} u+D_{2} v$.
Then we obtain the following system

$\left\{\begin{array}{l}\frac{\partial p}{\partial t}=\nabla \cdot(\nabla q-p \nabla q) \\ \frac{\partial q}{\partial t}=\nabla \cdot\left(-D_{1} D_{2} \nabla p+\left(D_{1}+D_{2}\right) \nabla q-q \nabla q\right)\end{array}\right.$

Since $u_{0}+v_{0} \equiv 1$ it is seen that $p \equiv 1$ solves the first equation of system (6). Based on the relation $v \equiv 1-u$ we obtain from (4) partial differential equation

$\left.\frac{\partial u}{\partial t}=\nabla \cdot\left[\left(D_{1}(1-u)+D_{2} u\right)\right) \nabla u\right]$.

Therefore, the implication $0 \leq u_{0} \leq 1 \Rightarrow 0 \leq u \leq 1$ follows easily from the maximum principle.

There are various numerical techniques for approximations of solutions of the ternary diffusion models (1)-(3). In particular, we refer to the papers $[16,17]$. The authors of [16] analyze the method of lines for diffusion equations. The paper [17] deals with iterative methods for this problem. The authors of both papers study stability and convergence of the constructed method in $L^{2}$ and in the Sobolev space $W^{1, \infty}$ . In this paper, as a tool for approximation of solutions to the initial boundary value problems for systems of equations obtained by an asymptotic expansion, we use a finite-difference methodology. In the construction of difference schemes we apply general ideas presented in [18, 19].

\section{Small Parameter Method}

Suppose that initial functions $u_{0}, v_{0}, w_{0} \in C^{2}(\bar{\Omega}, \mathbb{R})$ in (2) satisfy the relation $u_{0}+v_{0}+w_{0}=1$ for $x \in \bar{\Omega}$. In the sequel we assume that the diffusion coefficients $D_{1}, D_{2}, D_{3}$ in the system (1) satisfy the relation $D_{1}>D_{2}>D_{3}>0$. If $u, v, w$ satisfy (1)-(3), then $u+v+w \equiv 1$ and the system (1) reduces to the system of two equations

$\left\{\begin{array}{l}\frac{\partial u}{\partial t}=\nabla \cdot\left(D_{1} \nabla u-u\left[\left(D_{1}-D_{3}\right) \nabla u+\left(D_{2}-D_{3}\right) \nabla v\right]\right) \\ \frac{\partial v}{\partial t}=\nabla \cdot\left(D_{2} \nabla v-v\left[\left(D_{1}-D_{3}\right) \nabla u+\left(D_{2}-D_{3}\right) \nabla v\right]\right)\end{array}\right.$

It is more convenient to use the same change of variables $u, v$ to $p, q$ as in (5): $p=u+v, q=D_{1} u+D_{2} v$. For these functions we obtain the system of differential equations

$\left\{\begin{array}{l}\frac{\partial p}{\partial t}=\nabla \cdot[\nabla q-p \nabla(q-\varepsilon p)] \\ \frac{\partial q}{\partial t}=\nabla \cdot\left[-D_{1} D_{2} \nabla p+\left(D_{1}+D_{2}\right) \nabla q-q \nabla(q-\varepsilon p)\right]\end{array}\right.$

where $\varepsilon:=D_{3}$. Observe that if $\varepsilon=0$ then (7) is equivalent to (6). We expand the functions $p$ and $q$ in the series with respect to the small parameter $\varepsilon$ :

$p=p^{[0]}+\varepsilon p^{[1]}+\varepsilon^{2} p^{[2]}+\cdots=\sum_{l=0}^{\infty} \varepsilon^{l} p^{[l]}$,
$q=q^{[0]}+\varepsilon q^{[1]}+\varepsilon^{2} q^{[2]}+\cdots=\sum_{l=0}^{\infty} \varepsilon^{l} q^{[l]}$. 
Once we substitute these expansions to the system (7) and compare the coefficients we get the recurrence relation

$$
\left\{\begin{aligned}
\frac{\partial p^{[k]}}{\partial t}+ & \nabla \cdot\left(p^{[k]} \nabla q^{[0]}\right)=-\sum_{l=1}^{k-1} \nabla \cdot\left(p^{[l]} \nabla q^{[k-l]}\right) \\
& +\sum_{l=0}^{k-1} \nabla \cdot\left(p^{[l]} \nabla p^{[k-l-1]}\right) \\
\frac{\partial q^{[k]}}{\partial t}- & \left(D_{1}+D_{2}-q^{[0]}\right) \nabla^{2} q^{[k]}+2 \nabla q^{[0]} \cdot \nabla q^{[k]}=-D_{1} D_{2} \nabla^{2} p^{[k]} \\
& -\sum_{l=1}^{k-1} \nabla \cdot\left(q^{[l]} \nabla q^{[k-l]}\right)+\sum_{l=0}^{k-1} \nabla \cdot\left(q^{[l]} \nabla p^{[k-l-1]}\right)
\end{aligned}\right.
$$

for $k \geq 0$. We represent the initial conditions for the unknown functions $p^{[k]}$ and $q^{[k]}$ as follows:

$$
\begin{aligned}
& p^{[0]}(0, x)=\frac{u_{0}(x)+v_{0}(x)}{1-w_{0}(x)}=p_{0}(x) \sum_{l=0}^{\infty} w_{0}^{l}(x), \\
& q^{[0]}(0, x)=\frac{D_{1} u_{0}(x)+D_{2} v_{0}(x)}{1-w_{0}(x)}=q_{0}(x) \sum_{l=0}^{\infty} w_{0}^{l}(x),
\end{aligned}
$$

where $p_{0}=u_{0}+v_{0}, q_{0}=D_{1} u_{0}+D_{2} v_{0}$. It is easy to see that $p^{[0]}(0, x)=\frac{1-w_{0}(x)}{1-w_{0}(x)} \equiv 1$. If we compare the right-hand sides of (10) and (8), we obtain the initial conditions for the unknown coefficients $p^{[k]}, q^{[k]}$ for $k \geq 1$ :

$p^{[k]}(0, x)=-p_{0}(x) \frac{w_{0}^{k}(x)}{\varepsilon^{k}}, \quad q^{[k]}(0, x)=-q_{0}(x) \frac{w_{0}^{k}(x)}{\varepsilon^{k}}$

for $x \in \bar{\Omega}$.

Using a small parameter expansion of functions $p$ and $q$ we replace the original system of equations (7) by a separate systems of equations (9) for each pair of functions $\left(p^{[k]}, q^{[k]}\right)$, $k \geq 0$. The complexity of these systems is rising with increasing values of $k$. Therefore, the expansions (8) are practically limited to several terms. According to this we are mainly interested in the asymptotic nature of this truncated expansion for $\varepsilon \rightarrow 0, \varepsilon \ll 1$, then the possible divergence of the whole series is in general not important.

The study of asymptotic expansions was first made by Poincaré. According to Poincaré's definition, a series $\sum_{n=0}^{\infty} p^{[n]}(t, x ; \varepsilon) \varepsilon^{n}$ is said to be asymptotic to a function $p(t, x ; \varepsilon)$, i.e.

$p(t, x ; \varepsilon) \sim \sum_{n=0}^{\infty} p^{[n]}(t, x ; \varepsilon) \varepsilon^{n}$,

iff for every $N \geq 0$ and $\varepsilon \rightarrow 0, \varepsilon \ll 1$, we have

$$
p(t, x ; \varepsilon)-\sum_{n=0}^{N} p^{[n]}(t, x ; \varepsilon) \varepsilon^{n}=o\left(\varepsilon^{N}\right)
$$

This definition is equivalent to the following conditions

$$
\begin{aligned}
& \lim _{\varepsilon \rightarrow 0} \frac{p(t, x ; \varepsilon)}{\sum_{k=0}^{\infty} p^{[k]}(t, x ; \varepsilon) \varepsilon^{k}}=1 \quad \text { or } \\
& \lim _{\varepsilon \rightarrow 0} \frac{p(t, x ; \varepsilon)-\sum_{k=0}^{N} p^{[k]}(t, x ; \varepsilon) \varepsilon^{k}}{\varepsilon^{N}}=0 .
\end{aligned}
$$

Taking the first limit under consideration, we have

$\lim _{\varepsilon \rightarrow 0} \frac{p(t, x ; \varepsilon)}{\sum_{k=0}^{\infty} p^{[k]}(t, x ; \varepsilon) \varepsilon^{k}}=\frac{p(t, x ; 0)}{p^{[0]}(t, x ; 0)}$.

Since $D_{3}=\varepsilon \rightarrow 0$, the functions $p(t, x ; 0), p^{[0]}(t, x ; 0)$ become solutions of the same initial boundary value problem which leads to the conclusion that the limit is equal to 1 for every $N$. The same result we obtain for the expansion of $q(t, x ; \varepsilon)$. Therefore, the expansions (8) are asymptotic if only $\varepsilon \ll 1$. Even if the series do not converge, the asymptotic expansions give a good approximation of solutions if we take under consideration only few terms of these expansions with a very small value of $\varepsilon[11,20]$. This conclusion is confirmed by our numerical analysis in one dimensional case presented in Example 1. It turns out that our method is very effective for multidimensional couples, even in the case of complicated geometries.

\section{Numerical Analysis}

This section is devoted to a numerical analysis of solutions to the problem (9), (10), obtained by the small parameter expansion method. We construct a conservative difference scheme and present the numerical behavior of ternary solid solutions based on this method.

\subsection{Finite Difference Method}

We discretize the system of partial differential equations (9). Consider a uniform partition of $[0, T] \times \bar{\Omega}$, where $\bar{\Omega}=[-L, L]=\left[-L_{1}, L_{1}\right] \times \cdots \times\left[-L_{n}, L_{n}\right]$. Let $K \in \mathbb{R}$, $N_{1}, \ldots, N_{n}$ are positive integers and $N=\left(N_{1}, \ldots, N_{n}\right)$. Let $\left(h_{0}, h\right), h=\left(h_{1}, \ldots, h_{n}\right)$, stand for steps of a regular mesh on $[0, T] \times \bar{\Omega}$, such that $h_{0}=T / K$ and $h=L / N$. For $m=\left(m_{1}, \ldots, m_{n}\right) \in \mathbb{Z}^{n}$ we put $x^{(m)}=\left(m_{1} h_{1}, \ldots, m_{n} h_{n}\right)$ and

$\mathbb{R}_{h}^{n}=\left\{x^{(m)}: m \in \mathbb{Z}^{n}\right\}, \bar{\Omega}_{h}=\bar{\Omega} \cap \mathbb{R}_{h}^{n}$.

We convey that $\left(p_{h}^{[k]}, q_{h}^{[k]}\right):=\left(\left(p^{[k]}\right)_{h}^{(i, m)},\left(q^{[k]}\right)_{h}^{(i, m)}\right)$ will represent an approximation to the exact value of the functions $\left(p^{[k]}, q^{[k]}\right)$ at the point $\left(t_{i}, x_{m}\right)$ for $i \in\{0,1, \ldots, K\}$ and $m \in\{-N,-N+1, \ldots, N\}$.

Let $e_{j}=(0, \ldots, 1, \ldots 0) \in \mathbb{R}^{n}, 1 \leq j \leq n$, where 1 is the $j$ th coordinate. We employ standard and linear difference operators $\delta_{t}, \delta^{ \pm}=\left(\delta_{1}^{ \pm}, \ldots, \delta_{n}^{ \pm}\right), \delta^{(2)}=\left[\delta_{r j}\right]_{r j=1, \ldots, n}$ :

$\delta_{t} z=\frac{z^{(i+1, m)}-z^{(i, m)}}{h_{0}}$,

$\delta_{j}^{+} z=\frac{z^{\left(i, m+e_{j}\right)}-z^{(i, m)}}{h_{j}}, \quad \delta_{j}^{-} z=\frac{z^{(i, m)}-z^{\left(i, m-e_{j}\right)}}{h_{j}}$,

$\delta_{j j}^{(2)} z=\frac{z^{\left(i, m+e_{j}\right)}-2 z^{(i, m)}+z^{\left(i, m-e_{j}\right)}}{h_{j}^{2}}=\delta_{j}^{+} \delta_{j}^{-} z, \quad 1 \leq j \leq n$. 
Denote by $\nabla_{h}$ the discrete equivalent of the gradient operator $\nabla$. Let $\nabla_{h}^{2}=\sum_{j=1}^{n} \delta_{j j}^{(2)}$ then we define

$$
\begin{aligned}
& \nabla_{h}^{ \pm} \cdot\left(y \nabla_{h}^{\mp} z\right)=\sum_{j=1}^{n} \frac{1}{2}\left(\delta_{j}^{+} y \delta_{j}^{-} z+\delta_{j}^{-} y \delta_{j}^{+} z\right)+y \nabla_{h}^{2} z, \\
& \nabla_{h}^{ \pm} \cdot\left(y \nabla_{h}^{ \pm} z\right)=\sum_{j=1}^{n} \frac{1}{2}\left(\delta_{j}^{+} y \delta_{j}^{+} z+\delta_{j}^{-} y \delta_{j}^{-} z\right)+y \nabla_{h}^{2} z .
\end{aligned}
$$

With these conventions, the finite-difference scheme will be given by the following discrete system

$$
\left\{\begin{array}{c}
\delta_{t} p_{h}^{[k]}=-\nabla_{h}^{ \pm} \cdot\left(p_{h}^{[k]} \nabla_{h}^{\mp} q_{h}^{[0]}\right)-\sum_{l=1}^{k-1} \nabla_{h}^{ \pm} \cdot\left(p_{h}^{[l]} \nabla_{h}^{\mp} q_{h}^{[k-l]}\right) \\
\quad+\sum_{l=0}^{k-1} \nabla_{h}^{ \pm} \cdot\left(p_{h}^{[l]} \nabla_{h}^{ \pm} p_{h}^{[k-l-1]}\right) \\
\delta_{t} q_{h}^{[k]}=-D_{1} D_{2} \nabla_{h}^{2} p_{h}^{[k]}+\left(D_{1}+D_{2}\right) \nabla_{h}^{2} q_{h}^{[k]}-\sum_{l=0}^{k} \nabla_{h}^{ \pm} \\
\quad\left(q_{h}^{[l]} \nabla_{h}^{\mp} q_{h}^{[k-l]}\right)+\sum_{l=0}^{k-1} \nabla_{h}^{ \pm} \cdot\left(q_{h}^{[l]} \nabla_{h}^{ \pm} p_{h}^{[k-l-1]}\right)
\end{array}\right.
$$

with the discrete initial conditions

$\left(p_{h}^{[k]}\right)^{(0, m)}=p^{[k]}\left(0, x^{(m)}\right), \quad\left(q_{h}^{[k]}\right)^{(0, m)}=q^{[k]}\left(0, x^{(m)}\right)$.

The discrete Neumann boundary conditions have the form

$\left(p_{h}^{[k]}\right)^{(i, N+1)}=\left(p_{h}^{[k]}\right)^{(i, N-1)},\left(q_{h}^{[k]}\right)^{(i, N+1)}=\left(q_{h}^{[k]}\right)^{(i, N-1)}$,

$\left(p_{h}^{[k]}\right)^{(i,-N-1)}=\left(p_{h}^{[k]}\right)^{(i,-N+1)},\left(q_{h}^{[k]}\right)^{(i,-N-1)}=\left(q_{h}^{[k]}\right)^{(i,-N+1)}$,

for $i \in\{0, \ldots, K\}$.

\subsection{Numerical Simulations}

In this section we explore the capability of the constructed method (11)-(13) to provide good approximations to the exact solution of the PDE problem (1)-(3). We present some examples of tracer and up-hill diffusion in $\mathbb{R}^{1}$ and tracer diffusion in $\mathbb{R}^{2}$. Examples in one-dimensional case are similar to those presented in [16]. Therefore, one can compare the results of the method of lines obtained in [16] with the results obtained by our numerical scheme. We also provide more complicated two-dimensional cases which are absent from the literature.

Example 1 (Tracer at interface) Let $n=1$. In the numerical analysis we take $L=1$ and the diffusion parameters $D_{1}=0.18, D_{2}=0.08, D_{3}=\varepsilon$. The initial distribution $u_{0}$ is a small perturbation of the Heaviside function and $v_{0}$ is a symmetry of $u_{0}$ about the ordinate axis. The solution $w$ is initiated by a smoothed small peak

$$
\begin{aligned}
& u_{0}(x)=\Psi(100 x)-\Phi(100 x)+\mathbf{1}_{(0.01,1]}(x) \\
& v_{0}(x)=u_{0}(-x), \quad w_{0}(x)=2 \Phi(100 x),
\end{aligned}
$$

where $\mathbf{1}_{A}$ denotes the characteristic function of a set $A$, and

$$
\begin{aligned}
& \Psi(x)=\frac{-x^{3}+3 x+2}{4} \mathbf{1}_{[-1,1]}(x), \\
& \Phi(x)=\frac{\left(x^{2}-1\right)^{2}}{40} \mathbf{1}_{[-1,1]}(x)
\end{aligned}
$$

for $x \in[-1,1]$. We analyze the results for the PDE system (1) with the initial distributions given by (14) with respect to the small parameter $\varepsilon$. Illustrative simulations are provided with: $h_{0}=2 \cdot 10^{-5}$ and $h=2 \cdot 10^{-2}$. Based on formula (5) we can derive

$$
\begin{aligned}
& u^{[i]}=\frac{D_{2} p^{[i]}-q^{[i]}}{D_{2}-D_{1}}, \quad v^{[i]}=\frac{q^{[i]}-D_{1} p^{[i]}}{D_{2}-D_{1}}, \\
& w^{[i]}=1-u^{[i]}-v^{[i]}, \quad i=0, \ldots, k .
\end{aligned}
$$

We consider expansions (8) for $k=0,1,2$ :

- $k=0$, one term in each expansions: $p=p^{[0]}, q=q^{[0]}$. Numerical results for the respective $u^{[0]}, v^{[0]}, w^{[0]}$ calculated by (15) are presented in Fig. 1. Note that we obtain an expected behavior of densities $u, v, w$ regardless of the $\varepsilon$ value selection.

- $k=1$, two terms in each expansions: $p=p^{[0]}+\varepsilon p^{[1]}$, $q=q^{[0]}+\varepsilon q^{[1]}$. In Fig. 2 we observe the distributions of $u^{[1]}, v^{[1]}, w^{[1]}$ computed by (15) with different values of $\varepsilon$. Figure 3 presents the behavior of partial sums $u^{[0]}+\varepsilon u^{[1]}, v^{[0]}+\varepsilon v^{[1]}, w^{[0]}+\varepsilon w^{[1]}$ with different values of $\varepsilon$. Although oscillations of $u^{[1]}, v^{[1]}, w^{[1]}$ increase with decreasing values of $\varepsilon$, we obtain reasonable approximations of $u, v, w$ by means of partial sums $u^{[0]}+\varepsilon u^{[1]}, v^{[0]}+\varepsilon v^{[1]}, w^{[0]}+\varepsilon w^{[1]}$, respectively. Notice that these partial sums take values in the interval $[0,1]$ for all $\varepsilon$.

- $k=2$, three terms in each expansions: $p=p^{[0]}+\varepsilon p^{[1]}+\varepsilon^{2} p^{[2]}, \quad q=q^{[0]}+\varepsilon q^{[1]}+\varepsilon^{2} q^{[2]}$. Figure 4 presents the behavior of $u^{[2]}, v^{[2]}, w^{[2]}$ computed by (15). Numerical results of partial sums $u^{[0]}+\varepsilon u^{[1]}+\varepsilon^{2} u^{[2]}, v^{[0]}+\varepsilon v^{[1]}+\varepsilon^{2} v^{[2]}, w^{[0]}+\varepsilon w^{[1]}+$ $\varepsilon^{2} w^{[2]}$ are presented in Fig. 5. Similarly as in the case $k=1$, it can be observed that the oscillations of $u^{[2]}, v^{[2]}$, $w^{[2]}$ increase. Unlike the case $k=1$, the partial sums for $u, v, w$ take values in $[0,1]$ for sufficiently small $\varepsilon$. If $\varepsilon=0.01$, the partial sums for $u, v$ are not monotone. In Fig. 6 one can observe additional results for partial sums $u^{[0]}+\varepsilon u^{[1]}+\varepsilon^{2} u^{[2]}, v^{[0]}+\varepsilon v^{[1]}+\varepsilon^{2} v^{[2]}, w^{[0]}+\varepsilon w^{[1]}+$ $\varepsilon^{2} w^{[2]}$ at times $t=0.1$ and $t=0.2$ with fixed $\varepsilon=0.001$. We present these results to illustrate the dynamics of approximate distributions. 

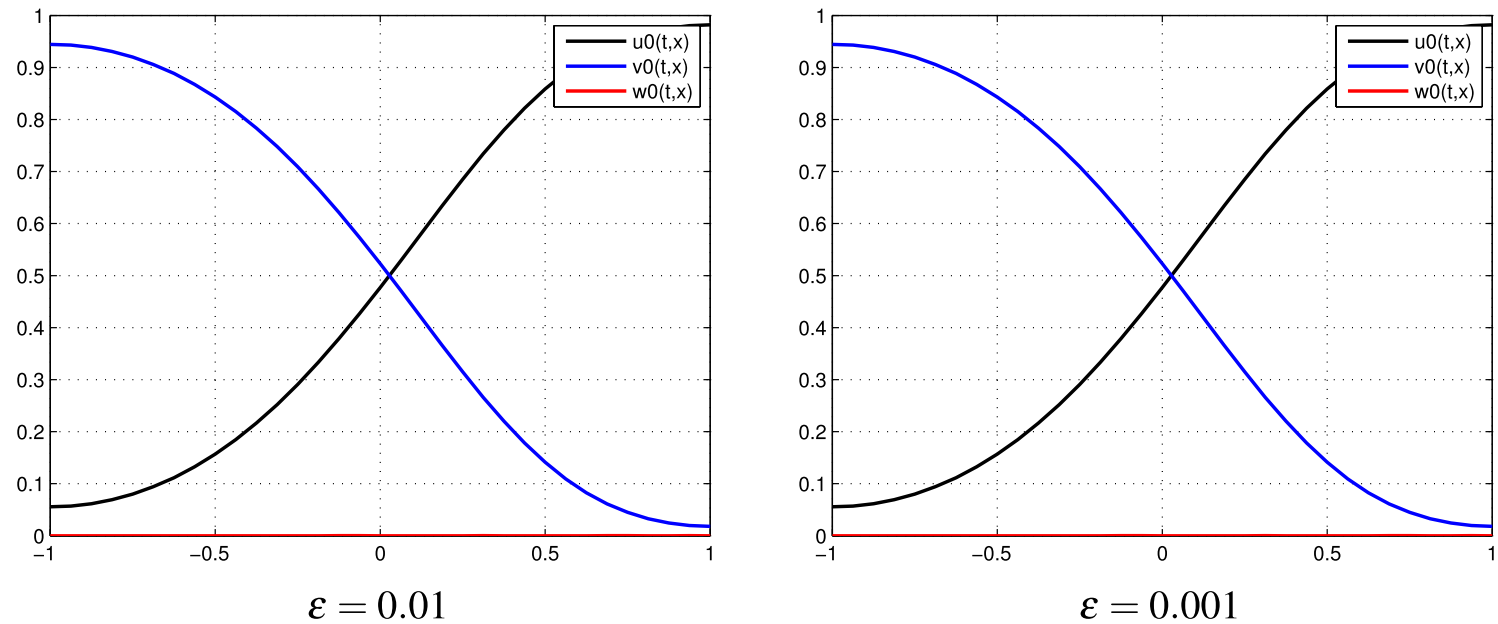

Fig. 1 (Tracer at interface) the case $k=0$ : functions $u^{[0]}, v^{[0]}, w^{[0]}$ for different values of $\varepsilon$ at time $t=0.8$

We conclude that the number of terms in the expansions (8) is closely related to the size of the parameter $\varepsilon$. Once we use more terms in the expansions, we need to decrease the value of $\varepsilon$ to get reasonable approximation of $u, v, w$ by means of respective partial sums, i.e. taking values in the interval $[0,1]$ and preserving monotonicity of partial sums for $u, v$.

Example 2 (Up-hill diffusion) We consider the onedimensional case and we set $L=1$ similarly as in Example 1. We take $D_{1}=0.2, D_{2}=0.08, D_{3}=0.001=\varepsilon$. We put some monotone functions as initial distributions of substances $u, v$ and we assume a constant initial distribution $w$ :

$u_{0}(x)=\Psi(100 x)+0.49 \mathbf{1}_{[-0.01,0.01]}(x)+0.98 \mathbf{1}_{(0.01,1]}(x)$
$v_{0}(x)=u_{0}(-x), \quad w_{0}(x)=0.02$

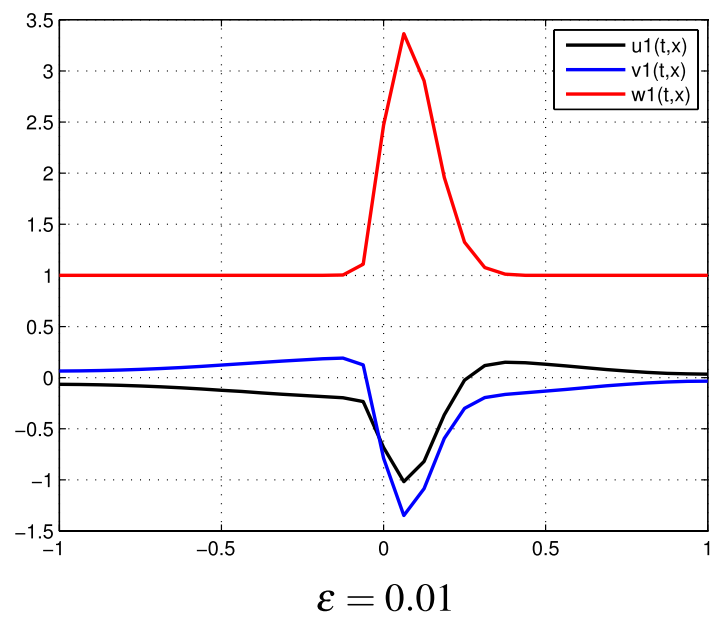

where

$\Psi(x)=\left(\frac{30}{176} x^{5}-\frac{50}{88} x^{3}+\frac{139}{176} x\right) \mathbf{1}_{[-1,1]}(x)$.

Results of numerical simulations for partial sums $u^{[0]}+\varepsilon u^{[1]}+\varepsilon^{2} u^{[2]}, v^{[0]}+\varepsilon v^{[1]}+\varepsilon^{2} v^{[2]}, w^{[0]}+\varepsilon w^{[1]}+\varepsilon^{2} w^{[2]}$ computed with $h_{0}=2 \cdot 10^{-5}, h=2 \cdot 10^{-2}$ are given in Fig. 7(b) at time $t=0.1$. Figure 7a presents initial functions $u_{0}, v_{0}, w_{0}$.

No numerical analysis for tracer diffusion in twodimensional case have been done so far in the literature because of the computational complexity, not to mention any efficient comparison of numerical results with reallife experiments. Due to our approach we constructed a numerical method which allows to perform computations

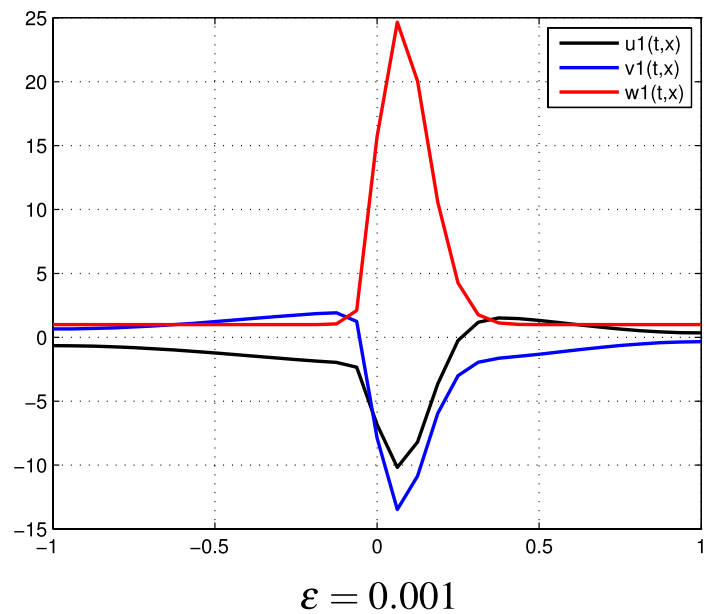

Fig. 2 (Tracer at interface) the case $k=1$ : functions $u^{[1]}, v^{[1]}, w^{[1]}$ for different values of $\varepsilon$ at time $t=0.8$ 

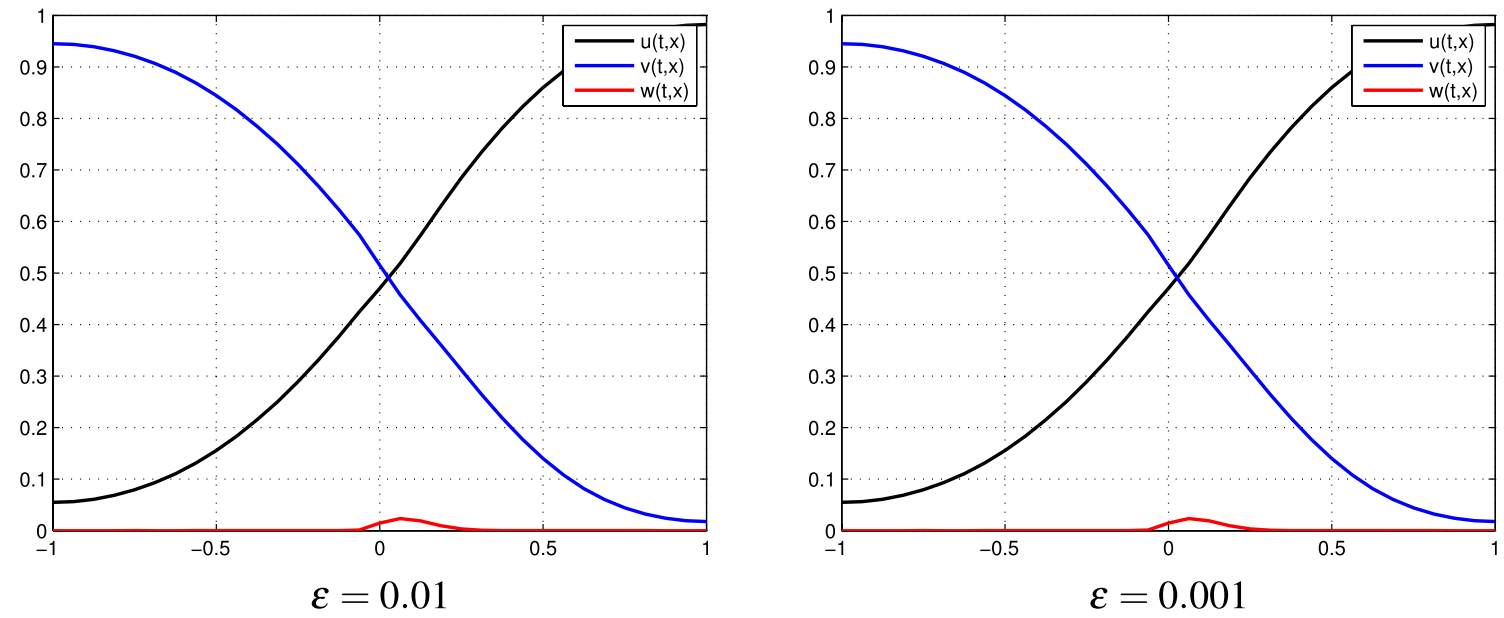

Fig. 3 (Tracer at interface) the case $k=1$ : partial sums $u^{[0]}+\varepsilon u^{[1]}, v^{[0]}+\varepsilon v^{[1]}, w^{[0]}+\varepsilon w^{[1]}$ for different values of $\varepsilon$ at time $t=0.8$
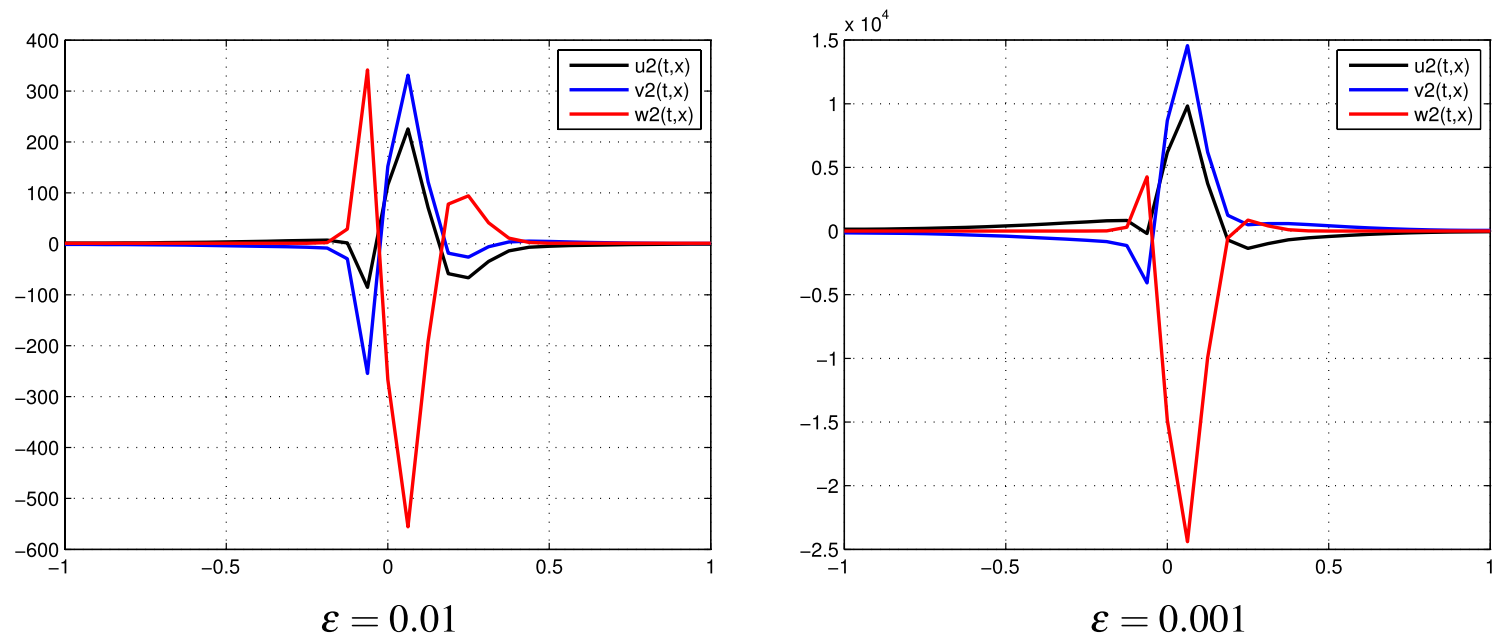

Fig. 4 (Tracer at interface) the case $k=2$ : functions $u^{[2]}, v^{[2]}, w^{[2]}$ for different values of $\varepsilon$ at time $t=0.8$
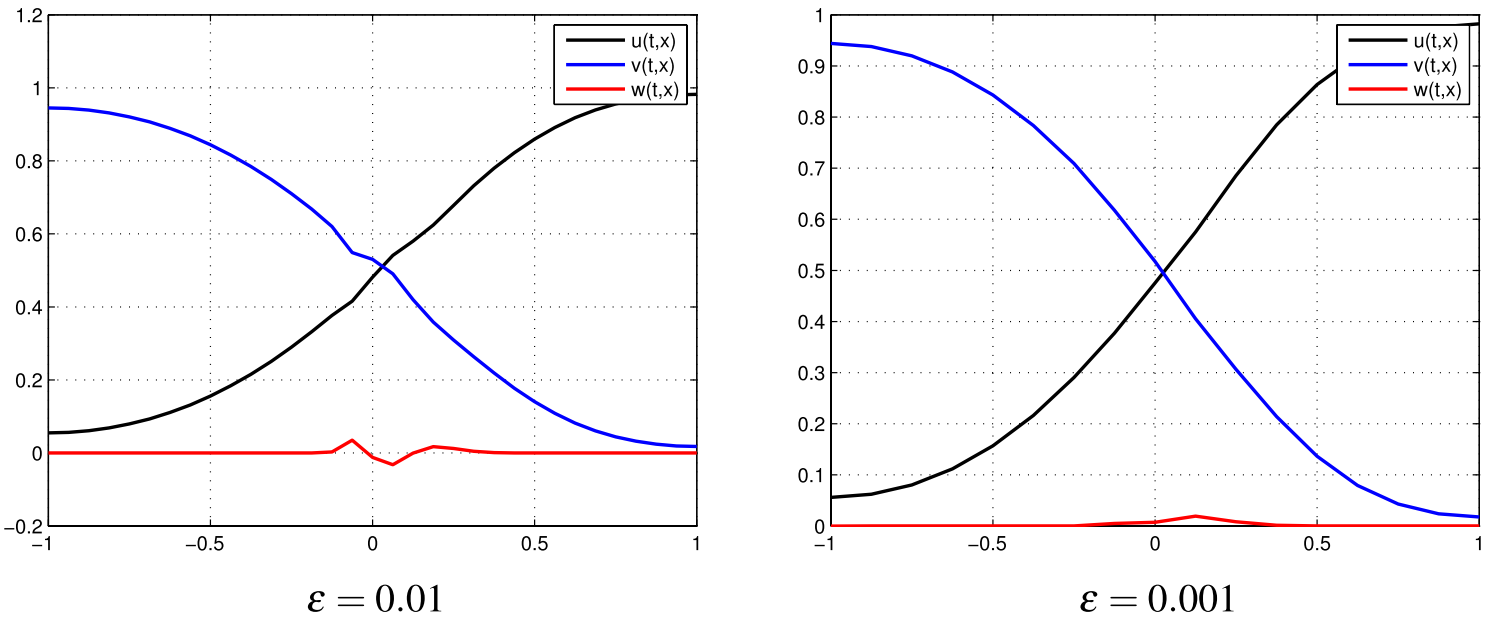

Fig. 5 (Tracer at interface) the case $k=2$ : partial sums $u^{[0]}+\varepsilon u^{[1]}+\varepsilon^{2} u^{[2]}, v^{[0]}+\varepsilon v^{[1]}+\varepsilon^{2} v^{[2]}, w^{[0]}+\varepsilon w^{[1]}+\varepsilon^{2} w^{[2]}$ for different values of $\varepsilon$ at time $t=0.8$ 

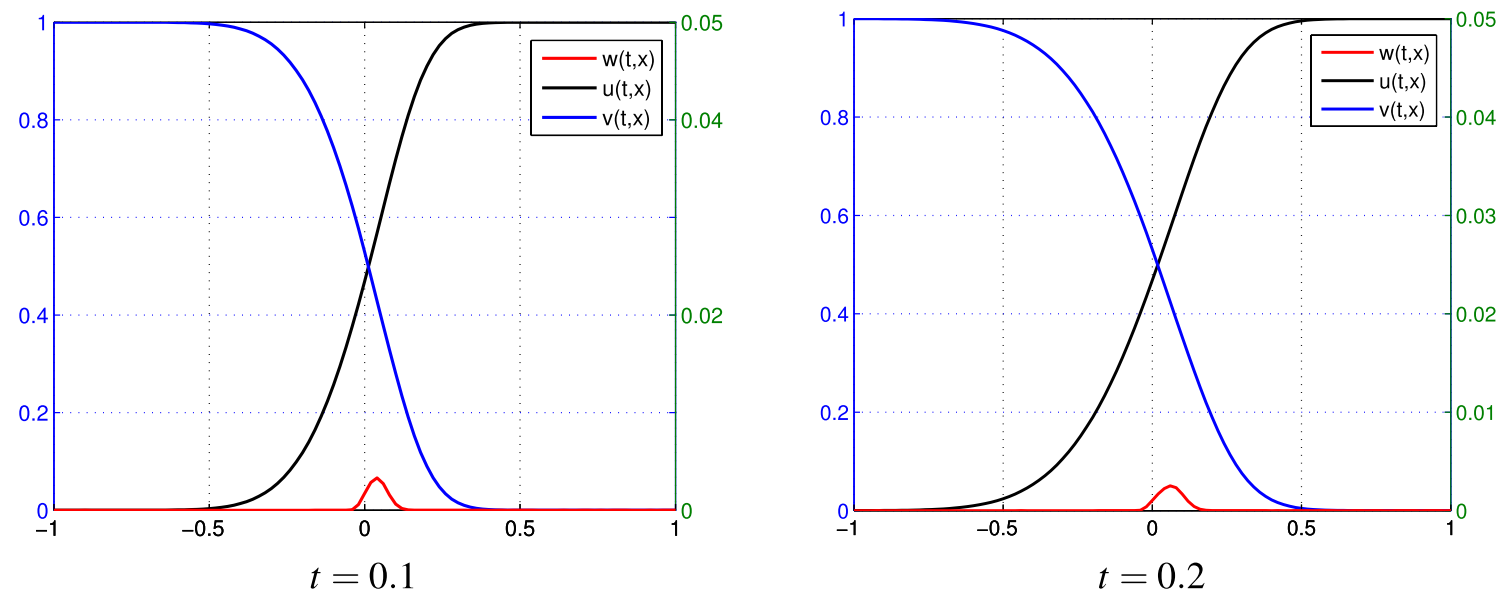

Fig. 6 (Tracer at interface) the case $k=2$ : partial sums for $u, v, w$ at time $t=0.1$ and $t=0.2$

even for multi-dimensional cases. We present two examples of numerical simulations for tracer diffusion in $\mathbb{R}^{2}$.

Example 3 (Tracer diffusion ) Consider the case $n=2$. Set $L=1$ and $D_{1}=0.2, D_{2}=0.08, D_{3}=0.001=\varepsilon$. Initial functions (Fig. 8) are given by

$u_{0}(x, y)=\theta(100 x)-\phi(100 x, 100 y)+\mathbf{1}_{(0.01,1]}(x)$,

$v_{0}(x, y)=u_{0}(-x, y), \quad w_{0}(x, y)=2 \phi(100 x, 100 y)$,

where

$\phi(x, y)=\frac{\left(x^{2}+y^{2}-1\right)^{2}}{100} \mathbf{1}_{(x \in[-1,1], y \in[-1,1])}(x, y) \mathbf{1}_{x^{2}+y^{2} \leq 1}(x, y)$,

$\theta(x)=\frac{-x^{3}+3 x+2}{4} \mathbf{1}_{[-1,1]}(x)$.

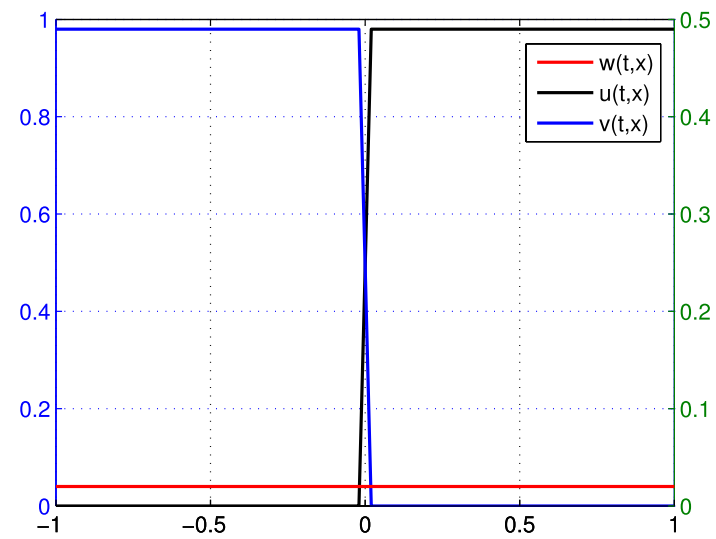

(a) Initial functions $(t=0)$
In Fig. 9a we observe approximate distributions of partial sums for $u, v, w$ with $k=2$ and the step sizes $h_{0}=2^{-15}$, $h=2^{-3}$ at time $t=0.2$. Figure $9 \mathrm{~b}$ presents enlargement of $w$.

In the above example initial distribution of $u$ and $v$ is symmetrical with respect to the plane $x=0$. Now we present two-dimensional example where the symmetry of initial distribution is broken.

Example 4 (Tracer diffusion) Consider the case $n=2$. Set $L=1$ and $D_{1}=0.15, D_{2}=0.08, D_{3}=0.001=\varepsilon$. Initial functions (Fig. 10) are given by

$$
\begin{aligned}
& u_{0}(x, y)=\theta\left(100\left(x-0.2 y^{2}\right)\right)-\phi(100 x, 100 y)+\mathbf{1}_{\left(0.01+0.2 y^{2}, 1\right]}(x), \\
& v_{0}(x, y)=\theta\left(-100\left(x-0.2 y^{2}\right)\right)-\phi(100 x, 100 y)+\mathbf{1}_{\left[-1,-0.01+0.2 y^{2}\right)}(x), \\
& w_{0}(x, y)=2 \phi(100 x, 100 y),
\end{aligned}
$$

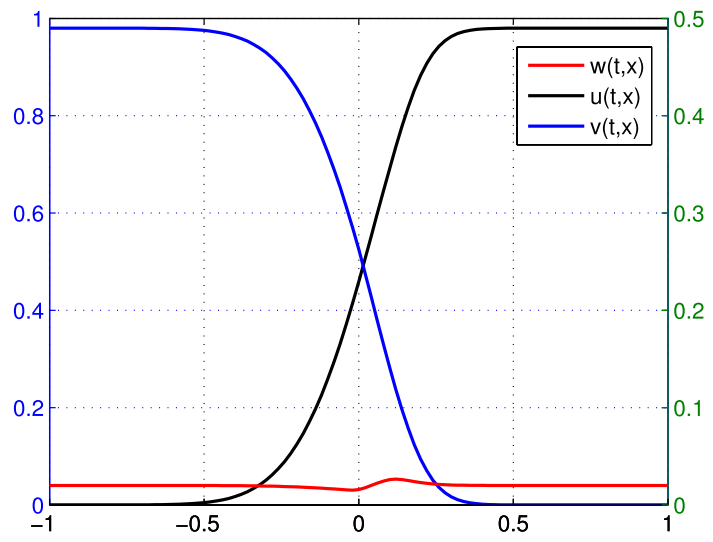

(b) $t=0.1$

Fig. 7 (Up-hill diffusion) a initial functions $u_{0}, v_{0}, w_{0}$, b partial sums $u^{[0]}+\varepsilon u^{[1]}+\varepsilon^{2} u^{[2]}, v^{[0]}+\varepsilon v^{[1]}+\varepsilon^{2} v^{[2]}, w^{[0]}+\varepsilon w^{[1]}+\varepsilon^{2} w^{[2]}$ at time $t=0.1$ 


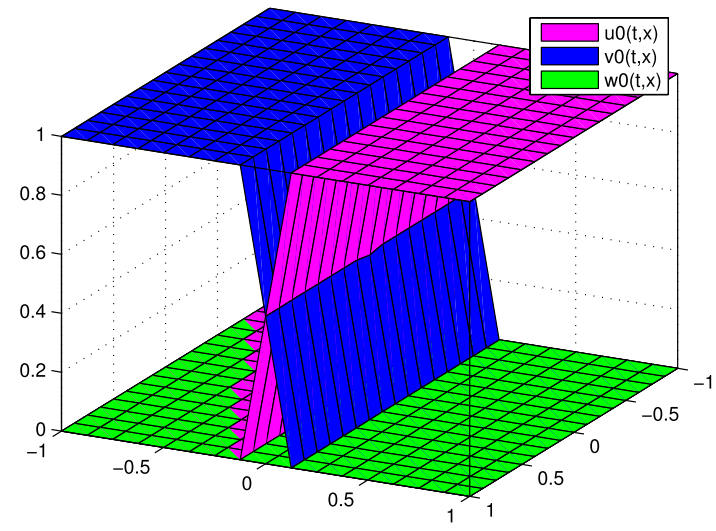

(a)

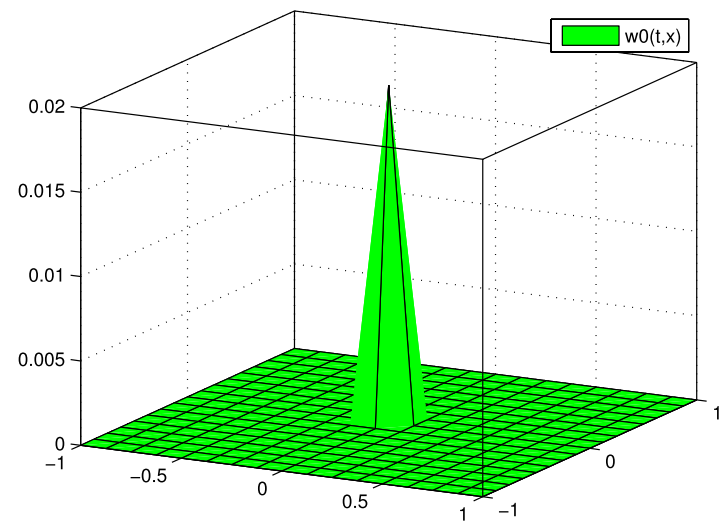

(b)

Fig. 8 (Tracer diffusion) initial distributions: a $u_{0}, v_{0}, w_{0}$, b enlargement of $w_{0}$

where $\phi$ and $\theta$ are the same as in Example 3. We perform simulations with parameters: the step sizes $h_{0}=2^{-15}$, $h=2^{-3}$ and the length of series expansions $k=2$. In Fig. 11(a) the approximate distributions of partial sums for $u, v, w$ at time $t=0.2$ are presented. Figure $11 \mathrm{~b}$ shows enlargement of $w$.

We conduct a brief analysis of marker positions in time, see [21]. By 'marker' we understand a small amount of substance placed in the diffusion couple, this substance moves according to locally not-balanced diffusive fluxes. Recall that diffusive fluxes are proportional to gradients of $u, v, w$. In the above tracer diffusion examples the marker is represented by the substance $w$. Referring to Example 1 the trajectory of the marker position is described by the ODE initial value problem

$\left\{\begin{array}{l}z^{\prime}(t)=v^{D}(t, z(t))=D_{1} u_{x}+D_{2} v_{x}+D_{3} w_{x} \\ z(0)=0\end{array}\right.$ where $v^{D}$ is the drift velocity. Since the diffusion coefficient $D_{3}$ is small, it can be omitted,

$v^{D}(t, z(t)) \approx q_{x}(t, z(t))=D_{1} u_{x}+D_{2} v_{x}$,

then the solution of the Cauchy problem (16) can be approximated by the solution of the following ODE problem

$\left\{\begin{array}{l}z^{\prime}(t)=q_{x}(t, z(t))=D_{1} u_{x}+D_{2} v_{x} \\ z(0)=0\end{array}\right.$

To solve the problems (16) and (17) numerically, we first use a polynomial fitting for discrete functions $u, v, w$ with respect to spatial variable. Then approximate solutions to the above ODE problems are computed by a second-order Runge-Kutta method. The results of computations with step sizes: $h_{0}=2 \times 10^{-5}, h=2 \times 10^{-2}$ are illustrated in Fig. 12. A mesh refinement leads to better accuracy, i.e. the solution to problem (17) gives a very good approximation

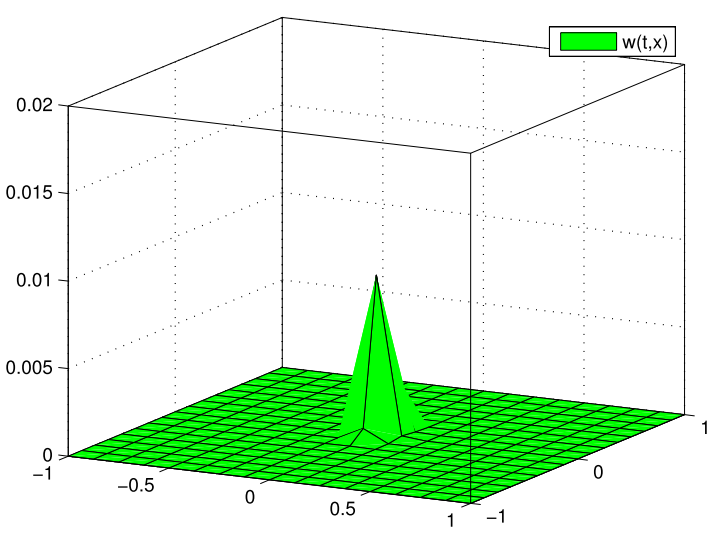

(b)

Fig. 9 (Tracer diffusion) partial sums with $k=2$ at time $t=0.2$ : a for $u, v, w$, b enlargement of $w$ 


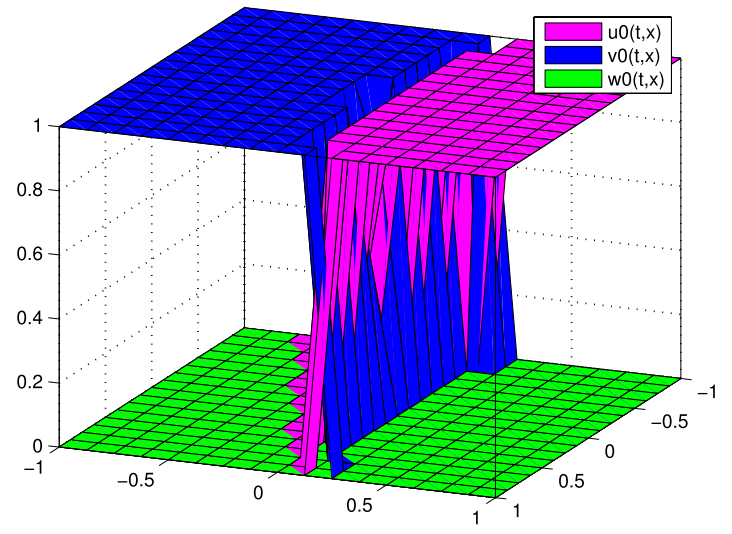

(a)

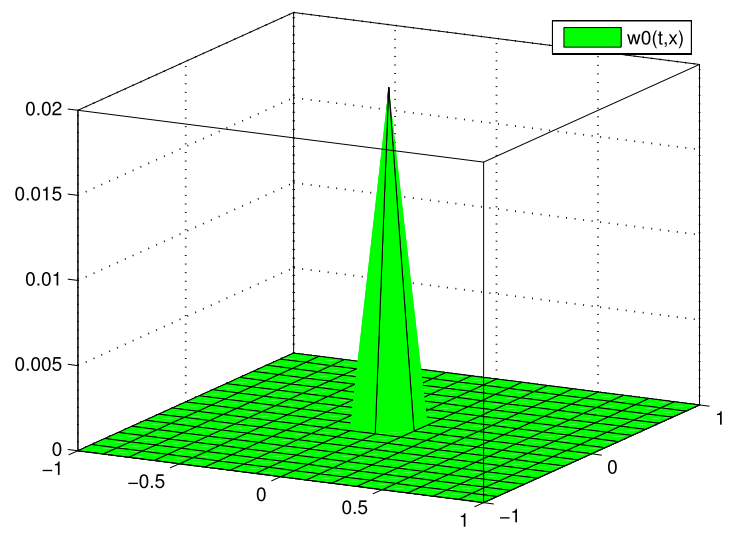

(b)

Fig. 10 (Tracer diffusion) initial distributions: $\mathbf{a} u_{0}, v_{0}, w_{0}, \mathbf{b}$ enlargement of $w_{0}$

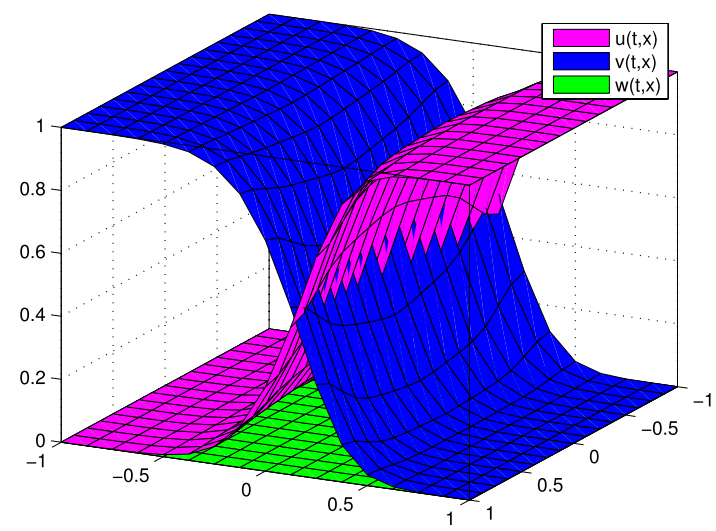

(a)

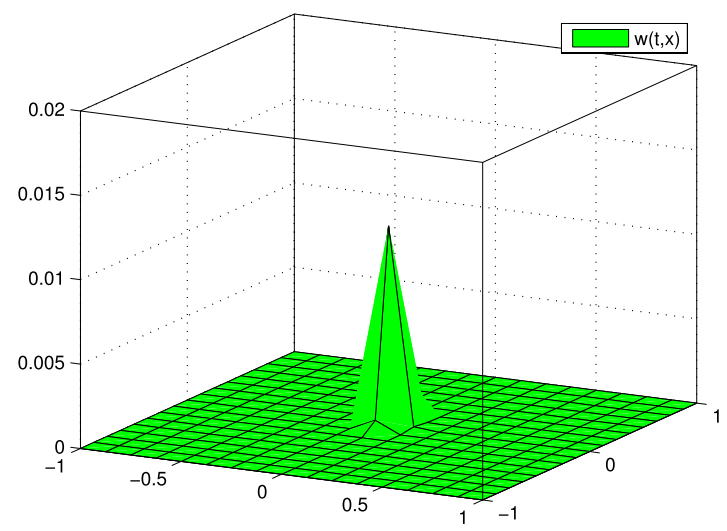

(b)

Fig. 11 (Tracer diffusion) partial sums with $k=2$ at time $t=0.2$ : a for $u, v, w$, $\mathbf{b}$ enlargement of $w$

of the solution to problem (16). An analogous discussion can be conducted for Examples 3 and 4.

\section{Conclusions and Remarks}

Before we close this paper with concluding remarks we would like to mention other applications of asymptotic expansion methods, in addition to those given in the introduction, to point out the importance of these techniques in a different branches of knowledge. Some applications can be observed in the fluid and solid mechanics theory. In [22] the asymptotic expansion method was used as a tool to derive a new two-dimensional shallow water model from the time-averaged non-dimensional Navier-Stokes equations. Asymptotic simplification procedures for linear and nonlinear wave propagation problems that contain large parameters are examined in [20]. A divergent asymptotic series found applications also in the boundary layer theory [23] and in the nonlinear wave theory [24].

We use the asymptotic expansion techniques to the ternary diffusion problem. Modeling of interdiffusion phenomena is not yet unified, and various methods differ from each other in the arbitrary choice of the reference velocity, i.e. the drift or convection velocity. In computational solid mechanics and physics it is defined as being equal to the mass average velocity [13]. In gases and fluids the drift is defined based on the volume average velocity [25]. It is still common to neglect drift (convection) and assume Fickian diffusion [26]. Such simplified approach to the mass transport was used there to a three-dimensional model of interdiffused quantum dots. The above methods do not allow considering all interdiffusion effects [26]. The use of widely accepted Onsager method $[27,28]$ is narrow by very limited data on transport coefficients. In material sciences and chemistry the drift definition bases on the Darken 


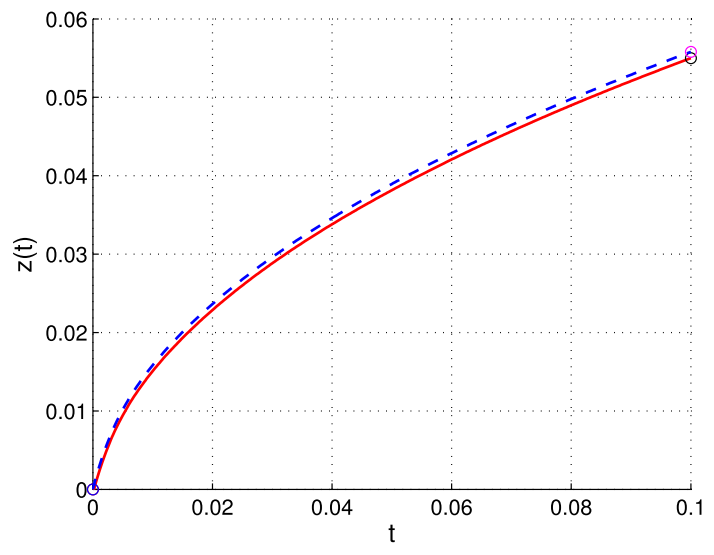

Fig. 12 The trajectory of marker position-approximate solutions of problems (16) (solid line) and (17) (dashed line) for $h_{0}=2 \cdot 10^{-5}, h=2 \cdot 10^{-2}$

method $[29,30]$. The Darken's basic postulate is that the reference velocity in multicomponent systems coincides with the drift velocity [30]. The drift in solids is often the vacancy flux generated during an interdiffusion process caused by the difference in the diffusion coefficients. The compatibility of Darken and Onsager formalisms has been already proved [31]. Onsager's and Fickian laws are known in life sciences, see [14].

In this paper we consider a strongly coupled system of diffusion equations with Neumann boundary conditions. This system is strongly coupled, highly nonlinear and possesses a parabolic type only in a very restricted subregion of the phase space, which is related to the mass conservation. This causes analytical and numerical difficulties. To overcome these difficulties we use asymptotic expansions of solutions in divergent series with respect to a small parameter $\varepsilon:=D_{3}$. We obtain another system of equations which is more convenient to analyze. Our examples described in the previous section are based on this method and illustrate the behavior of ternary solid solutions (alloys), in particular: up-hill diffusion in $\mathbb{R}^{1}$, tracer diffusion in $\mathbb{R}^{1}$ and tracer diffusion in a complex geometry in $\mathbb{R}^{2}$. We observe that solutions of the new system obtained by the expansion method with several terms, e.g. $k=1$ or $k=2$, reflect an expected behavior of solutions of the original diffusion problem. There are many open questions in the theory and practice of small parameter expansions:

- Whether or not it is possible to extend the small parameter method onto the case of singularities [11]? In the case of ternary diffusion problems singularities may be caused by irregularity of initial distributions $u, v, w$, e.g. Heaviside or smoothed peak type functions.

- Is it possible to apply the small parameter method to a derivation of interfaces, e.g. via Matano's method [32]?
- If indeed replacing Neumann boundary conditions by Stefan free boundary conditions is easy to conduct?

- Is it possible to generalize our method onto the case of media with variable diffusion coefficients and multiphase media?

- Is it possible to formulate and perform computations by means of a small parameter method within Onsager's formalism? [33-35]

Acknowledgements This work was supported by the Polish Science Center Decision No. DEC-2011/02/A/ST8/00280.

Open Access This article is distributed under the terms of the Creative Commons Attribution 4.0 International License (http:// creativecommons.org/licenses/by/4.0/), which permits unrestricted use, distribution, and reproduction in any medium, provided you give appropriate credit to the original author(s) and the source, provide a link to the Creative Commons license, and indicate if changes were made.

\section{References}

1. Tucciarone $\mathbf{J}$ (1973) The develompent of the theory of summable divergent series from 1880 to 1925 . Arch Hist Exact Sci 10:1-40

2. Ferraro G (1999) The first modern definition of the sum of a divergent series: an aspect of the rise of 20th century mathematics. Arch Hist Exact Sci 54:101-135

3. Dyson DJ (1952) Divergence of perturbation theory in quan tum electrodynamics. Phys Rev 85:32-33

4. Fischer J (1997) On the role of power expansions in quantum field theory. Int J Mod Phys A 12:3625-3663

5. Suslov IM (2005) Divergent perturbation series. J Exp Theor Phys 100:1188-1234

6. Aniceto I, Russo JG, Schiappa R (2015) Resurgent analysis of localizable observables in supersymmetric gauge theories. J High Energy Phys JHEP 03:172

7. Rivasseau V, Wang Z (2014) How to resum Feynman graphs. Ann Henri Poincaré 15:2069-2083

8. Zinn-Justin J (2010) Summation of divergent series: orderdependent mapping. Appl Numer Math 60:1454-1464

9. Caliceti E, Meyer-Hermann M, Ribeca P, Surzhykov A, Jentschura UD (2007) From useful algorithms for slowly convergent series to physical predictions based on divergent perturbative expansions. Phys Rep 446:1-96

10. Dunne GV, Ünsal M (2014) Generating nonperturbative physics from perturbation theory. Phys Rev D 89:041701(R)

11. Banasiak J, Lachowicz M (2014) Methods of small parameter in mathematical biology. Birkhäuser, Boston

12. Archibasov AA, Korobeinikov A, Sobolev VA (2015) Asymptotic expansions of solutions in a singularly perturbed model of virus evolution. Comput Math Math Phys 55:240-250

13. Feulvarch E (2012) Finite element modeling of interdiffusion phenomena in solid metals. Competes Rendus Mec 340:695-701

14. Thai RC, Chang HK (1979) A mathematical study of non-equimolar ternary gas diffusion. Bull Math Biol 41:591-606

15. Kucza W, Danielewski M (2006) Electrodiffusion in nanostructures. e J Surf Sci Nano Tech 4:464-467

16. Leszczyński H, Matusik M (2014) The method of lines for ternary diffusion problems. Abstract Appl Anal 2014:517285 
17. Leszczyński H, Wrzosek M (2014) Iterative methods for ternary diffusions. Bound Value Probl 2014:87. doi:10.1186/1687-2770-2014-87

18. Samarskii AA (2001) The theory of difference schemes. Vol. 249 of monographs and textbooks in pure and applied mathematics, Marcel Dekker, New York, NY, USA

19. Samarskii AA, Matus PP, Vabishchevich PN (2002) Difference schemes with operator factors. Vol. 546 of mathematics and its applications. Kluwer Academic Publisher, Dordrecht

20. Zauderer E (2006) Partial differential equations of applied mathematics. Wiley, New Jersey

21. Danielewski M, Leszczyński H (2015) Computation of trajectories and displacement fields in a three-dimensional ternary diffusion couple: parabolic transform method. Math Probl Eng 650452:11. doi:10.1155/2015/650452

22. Rodriguez JM, Taboada-Vazquez R (2015) Time-averaged shallow water model: Asymptotic derivation and numerical validation. J Math Anal Appl 428:930-950

23. Cousteix J, Mauss J (2007) Asymptotic analysis and boundary layers. Springer, Berlin

24. Shen SS (2012) A course on nonlinear waves. Springer, Netherlands

25. Brenner H (2012) Beyond Navier-Stokes. Int J Eng Sci 54:67-98

26. Gunawan O, Djie HS, Ooi BS (2005) Electronics states of interdiffused quantum dots. Phys Rev B 71:205-319

27. De Groot SR (1951) Thermodynamics of irreversible processes. Chap. I, Sec. 2, pp 5-9. North-Holland Publishing Comp., Amsterdam
28. Hirschfelder JO, Curtiss CF, Bird RB (1954) Molecular theory of gases and liquids. Chap. 11, Sec. 2a. Wiley, New York, pp 705-708

29. Danielewski M, Filipek R, Holly K, Bożek B (1994) Interdiffusion in multicomponent solid solutions, the mathematical model for thin films. Phys Status Solidi (a) 145:339-350

30. Darken LS (1948) Trans AIME 174:184-201

31. Bożek B, Danielewski M, Tkacz-Śmiech K, Zajusz M (2015) Interdiffusion: compatibility of Darken and Onsager formalisms. Mater Sci Technol 31:1633-1641. doi:10.1179/17432847 15Y.0000000077

32. Danielewski M, Leszczyński H (2015) Generalization of Matano's method: interdiffusion in solutions with volume change. Int J Math Anal 9(30):1463-1476

33. Danielewski M, Gusak A, Bożek B, Zajusz M (2016) Model of diffusive interaction between two-phase alloys with explicit finetuning of the morphology evolution. Acta Mater 108:68-84

34. Esham BF Jr (1991) Galerkin methods for a singularly perturbed hyperbolic problem with nonlocal nonlinearity. Comput Math Appl 22:1-22

35. Schmidt K, Hiptmair R (2015) Asymptotic boundary element methods for thin conduction sheets. Discrete Cont Dyn Syst Ser S 8:619-647 